\title{
Nucleation and evaporation of domains due to electric field at room temperature in $\mathrm{BaTiO}_{3}$ single crystals
}

\author{
C M DUDHE* and P R ARJUNWADKAR \\ Department of Physics, Government Institute of Science, Nagpur 440 001, India
}

MS received 25 August 2014; accepted 30 April 2015

\begin{abstract}
A study of nucleation and evaporation of $90^{\circ}$ and $180^{\circ}$ domains by external direct current (dc) electric field at room temperature in barium titanate single crystals has been carried out using reflecting microscope. It was observed that both the $90^{\circ}$ and $180^{\circ}$ domains were nucleated at some sites, while evaporated at some other sites of the crystal surface. The $90^{\circ}$ domain follows the mechanism of micro-domain wall nucleation as well as the evaporation based on impurity dipoles, like reported for $\mathrm{KNbO}_{3}$. The mechanism of the $180^{\circ}$ domain nucleation is not established yet. However, in both cases, nucleation and evaporation are operative simultaneously by the same electric field, which seems to be quite interesting and which can be attributed to the different critical lengths of the domains.
\end{abstract}

Keywords. Impurity dipoles; domain walls; nucleation; evaporation; spontaneous polarization.

\section{Introduction}

Barium titanate $\left(\mathrm{BaTiO}_{3}\right)$ is a well-known ferroelectric material. This material is highly investigated for various possible applications. ${ }^{1-3}$ Above $120^{\circ} \mathrm{C}$ temperature, $\mathrm{BaTiO}_{3}$ has the cubic perovskite structure. Below this temperature it remains in tetragonal phase up to $5^{\circ} \mathrm{C}$ having polar axis $(0,0,1)$. In this phase the $\mathrm{Ti}$ and $\mathrm{O}$ ions move relative to $\mathrm{Ba}$ at the origin from their cubic positions, Ti at $(1 / 2,1 / 2$, $1 / 2)$, and three oxygen at $(1 / 2,1 / 2,0),(1 / 2,0,1 / 2)$ and $(0,1 / 2,1 / 2)$, to $\mathrm{Ti}$ at $\left(1 / 2,1 / 2,1 / 2+d z_{\mathrm{Ti}}\right), \mathrm{O}_{\mathrm{i}}$ at $(1 / 2,1 / 2$, $\left.d z_{\mathrm{O}_{\mathrm{i}}}\right), \mathrm{O}_{\mathrm{ii}}$ at $\left(1 / 2,0,1 / 2+d z_{\mathrm{Oii}_{\mathrm{i}}}\right)$ and $\left(0,1 / 2,1 / 2-d z_{\mathrm{O}_{\mathrm{ii}}}\right)$. The shifting of oxygen octahedron is very little distorted by $\mathrm{O}^{-}$ions displacement and no large change occurs in $\mathrm{Ba}-\mathrm{O}$ bond length, but the $\mathrm{Ti}-\mathrm{O}$ bond length is markedly altered, which constitutes Ti-O dipoles. ${ }^{4}$

In addition to Ti-O dipoles, impurity dipoles are present in the crystals as impurities are always the part of ferroelectric materials, grown by the flux melt method. In the present study, the crystals were grown by the wellknown Remeika's method in platinum crucible at high temperature of around $1200^{\circ} \mathrm{C}$. At such high temperature, platinum impurities are introduced very easily into the melt. ${ }^{5}$ Moreover, such crystals are oxygen deficient. ${ }^{6,7}$ Consequently, an impurity dipole will be resulted, if the oxygen vacancy exists at the site of octahedron, the impurity ion being the positive pole and vacant oxygen site being the negative pole of the impurity dipole. $^{8}$

\footnotetext{
*Author for correspondence (chandraguptadudhe@gmail.com)
}

Several investigations have been made to investigate domain structures, domain nucleation, sidewise motion of domain walls, the domain-dislocation interaction and the impurity dipole-domain interaction. ${ }^{5,9-12}$ Potnis et $a l^{13}$ reported an excellent review of progress in the field of domain modelling and domain imaging techniques of ferroelectrics. Nowadays various advanced techniques such as atomic force microscopy (AFM), scanning electron microscopy (SEM), transmission electron microscopy (TEM), X-ray diffraction, second harmonic generation microscopy (SHGM), etc. are being used to investigate the domain structure. In recent times, Dudhe et $a l^{14}$ used TEM to investigate nanodomain structures in $\mathrm{BaTiO}_{3}$ nanoparticles. A good amount of literature on the domain morphology by using simple metallurgical reflecting microscope and possible mechanisms of domain wall nucleation is widely available due to Ingle et $a .^{8,12,15-17}$ Ingle and Moon $^{8}$ reported a possible mechanism of domain walls nucleation in $\mathrm{KNbO}_{3}$ single crystals. The mechanism proposes that the impurity dipoles tend to orient themselves in the direction of spontaneous polarization $P_{\mathrm{s}}$ of the crystal when the crystal is cooled down below the Curie temperature. However, if the orientation of the dipoles is different than that of $P_{\mathrm{s}}$ of the crystal then strain will be developed at that site and, in order to reduce this strain, a pair of domain walls will be nucleated during the paraelectric-ferroelectric phase transition such that the spontaneous polarization $P_{\mathrm{s}}$ at that site coincides the orientation of the impurity dipoles. Such nucleation of domains is possible below the Curie temperature under the influence of an external electric field. Further, it is also proposed that the mechanism of evaporation of 
domain walls is just reversed to that of the nucleation. ${ }^{17}$ Accordingly, the reverse field for the evaporation should be 1.5 times to that of required field for the nucleation.

Although these models are better suited for the $90^{\circ}$ domains independently for the nucleation and evaporation of domains, no explanation is given for the case when both are occurring simultaneously. Moreover, not much attention is given to the nucleation and evaporation of the $180^{\circ}$ domains. In the present study, Ingle's model $^{15,17}$ was applied to simultaneously occurring nucleation and evaporation of domains in $\mathrm{BaTiO}_{3}$ crystals and results are interpreted.

\section{Experimental}

$\mathrm{BaTiO}_{3}$ single crystals were grown by a well-known Remeika's flux method. The grown crystals have a smooth cleavage along the pseudocubic (001) plane; therefore, they were smoothly cut to get (001) surface (c-plates) without disturbing domain structure of the crystals. These c-plates were observed under the metallurgical reflecting microscope. Suitable site of the surface of the c-plate sample containing the $90^{\circ}$ domains was photographed by using microscope-attachment-camera system. For the $180^{\circ}$ domains, the c-plate sample was etched by hydrochloric acid for few seconds, and was photographed using the same system. A suitable direct current (dc) electric field $E$ was applied perpendicular to the major surface (c-plate) of the sample for a certain period, washed with methanol and same site was again photographed.

\section{Results and discussion}

\subsection{Nucleation and evaporation of the $90^{\circ}$ domains}

Figure 1 shows photomicrograph of pesudocubic (001) surface of the $\mathrm{BaTiO}_{3}$ crystal. Two sets of the $90^{\circ}$ domain walls are clearly observed, one set is parallel to the marked line CD and the other is parallel to marked line AB. Schematically they are shown in figure $2 a$ and $b$, respectively, where the outside marked $P_{\mathrm{s}}$ is the directions of spontaneous polarization of the crystal as a whole, while the inside marked $P_{\mathrm{s}}$ is the direction of spontaneous polarization of independent domains present at that site (a circle with dot represents the direction of polarization perpendicular to the observed plane). On critical examination, it is concluded that the domain walls parallel to line $\mathrm{CD}$ are a-domains (where the $P_{\mathrm{s}}$ always lies on the plane) and the domain walls parallel to line $\mathrm{AB}$ are c-domains (where $P_{\mathrm{s}}$ lies alternatively on the plane and into the plane).

Same crystal surface after the application of electric field of about $5 \mathrm{kV} \mathrm{cm}^{-1}$ for $15 \mathrm{~min}$ is shown in figure 3 . This photomicrograph shows that the set of domains

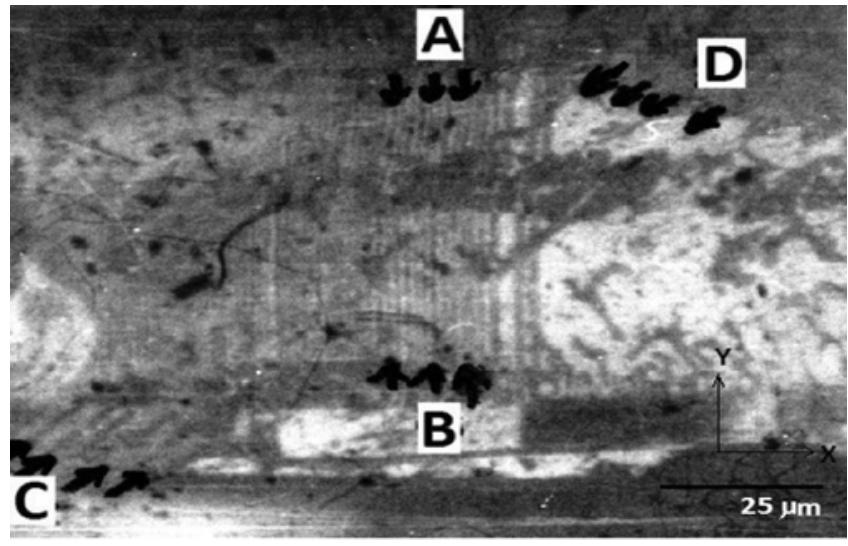

Figure 1. Photomicrograph of (001) surface of the crystal showing two set of domain walls, one parallel to $A B$ line and the other parallel to CD line $(450 \times)$.
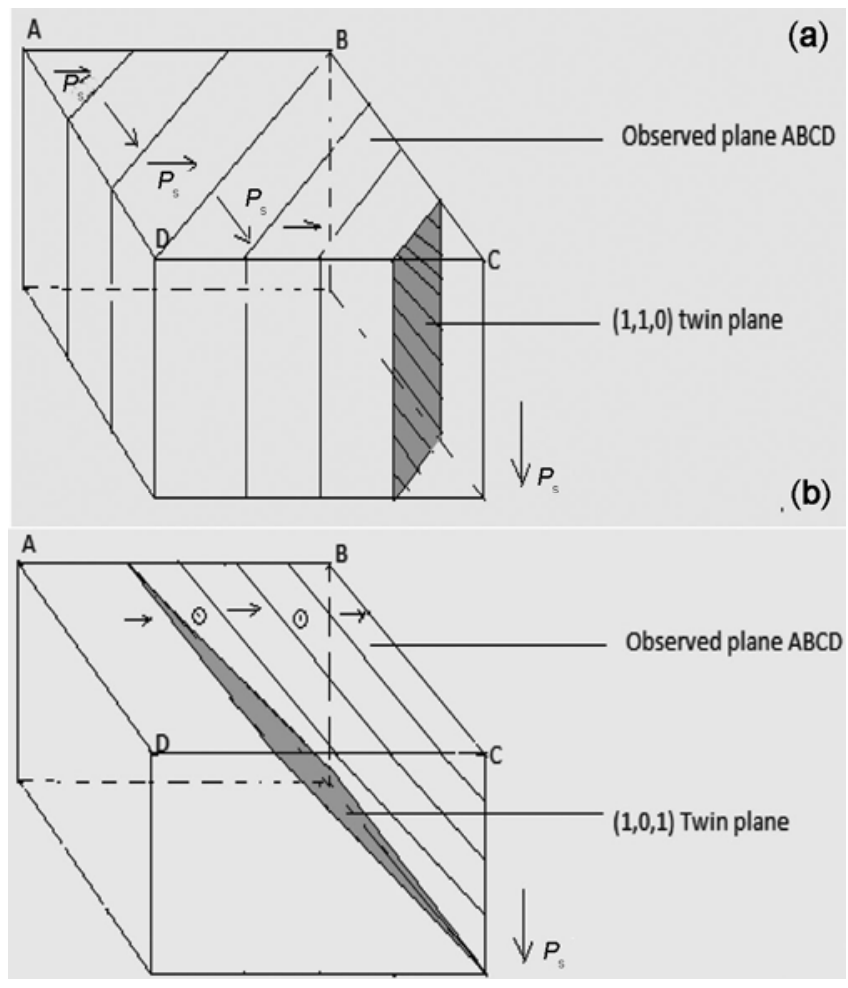

Figure 2. Schematic sketch of (a) a-b types $90^{\circ}$ domains parallel to $\mathrm{CD}$ line and (b) a-c types $90^{\circ}$ domains parallel to $\mathrm{AB}$ line observed in figure 1 .

parallel to line CD is almost removed by the electric field due to evaporation, whereas a new line marked by $\mathrm{MN}$ seems to be appeared parallel to line AB. The appearance of this line indicates a process reverse to the process of evaporation, hence the marked line $\mathrm{MN}$ is said to be a nucleated domain wall.

The appearance of a line $\mathrm{MN}$ can be explained by the principle of the $90^{\circ}$ domain wall nucleation by impurity dipoles, and by using the schematically drawn 


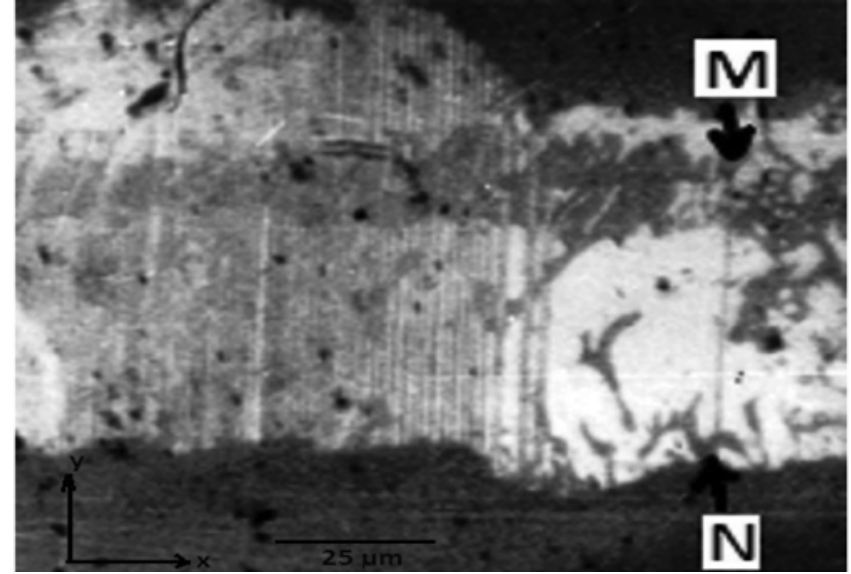

Figure 3. Photomicrograph of same surface (in figure 1) after application of $\mathrm{dc}$ field $5 \mathrm{kV} \mathrm{cm}{ }^{-1}$ for 15 min showing the appearance of a new domain wall MN (450×).

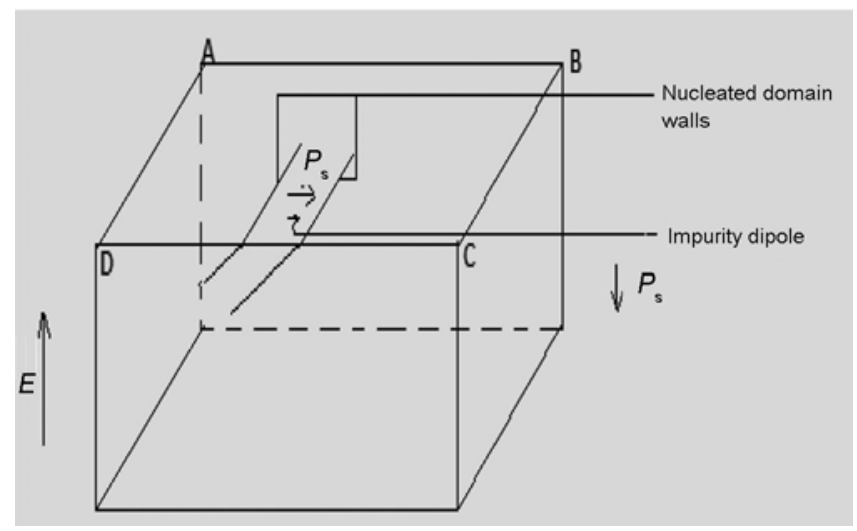

Figure 4. Schematic representation of principle of $90^{\circ}$ microdomain walls nucleation.

figure (figure 4). The applied electric field may cause the excitation of impurity ions and form impurity dipoles. ${ }^{16}$ These dipoles will try to orient themselves along the direction $P_{\mathrm{s}}$ of the crystal; if they fail, a strain is developed in the crystal at that site. To reduce this strain, a pair of microdomain walls is nucleated with the new spontaneous localized polarization $P_{\mathrm{s}}$ at that site. If there is a co-operative arrangement of the dipoles then microdomains join each other in head-to-tail manner and domain walls are formed. MN line in figure 3 is one of such nucleated $90^{\circ}$ domain walls. However, nucleation of single wall instead of pair is surprising. The single line $\mathrm{MN}$ may be attributed to an unresolved pair of domain walls where the thickness of the wall might be of the order of nanometre ${ }^{9}$ or it is an exception to the principle of domain wall nucleation, or some other mechanism might be operative in this case.

Simultaneous de-excitation of impurity dipoles was seen, for the same applied electric field, at some another site of the observed plane, resulting in evaporation of domain walls. For example, the applied electric field acts as a reversed field for the domain walls parallel to $C D$ line (in figure 1), giving rise to reverse strain in the crystal. The domain wall energy is then used to reduce the strain at that site. Consequently, the walls parallel to line $\mathrm{CD}$ are evaporated (see figure 4 ) and dipoles relaxed in to ionic impurities.

Quantitatively nucleation of domains can be explained by using equation suggested by Ingle et al. ${ }^{15}$ According to the study the critical length of nucleation of the $90^{\circ}$ domain is

$$
l_{\mathrm{cr}}=\frac{8 \alpha}{3 P_{\mathrm{s}}\left(E-E_{\mathrm{cr}}\right)},
$$

where $\alpha$ is the domain wall energy, $P_{\mathrm{s}}$ the spontaneous polarization, $E$ the applied electric field and $E_{\mathrm{cr}}$ the critical electric field for nucleation of domains. By assuming the minimum critical electric field $\left(E_{\mathrm{cr}}\right)$ which is about $60 \mathrm{~V} \mathrm{~cm}^{-1}$ for the switching of the $90^{\circ}$ domains in $\mathrm{BaTiO}_{3}$ single crystals, ${ }^{18}$ which looks to be negligible as compared to the applied field $\left(5 \mathrm{kV} \mathrm{cm}^{-1}\right)$, expression (1) can be written as

$$
l_{\mathrm{cr}} \sim \frac{8 \alpha}{3 P_{\mathrm{s}} E} .
$$

Using optimum values of $P_{\mathrm{s}}$ as $26 \mu \mathrm{C} \mathrm{cm}^{-2}$ and the $90^{\circ}$ domain wall energy $(\alpha) \sim 80 \mathrm{erg} \mathrm{cm}^{-2}$ as given in the literature ${ }^{19}$ the critical length was estimated to be $\sim 1.7 \mu \mathrm{m}$. This implies that the domain wall nucleated by $5 \mathrm{kV} \mathrm{cm}^{-1}$ must have a length more than $1.7 \mu \mathrm{m}$ to form a sustainable domain structure. The length of nucleated domain MN (figure 3) is about $50 \mu \mathrm{m}$, indicating that it is formed by joining many microdomains each of which length is higher than $1.7 \mu \mathrm{m}$. This is possible only when there is a head-to-tail cooperative arrangement of the dipoles.

Similarly from the kinetic process of evaporation, ${ }^{17}$ it is found that

$$
E^{\prime} \sim \frac{3}{2 E},
$$

where $E^{\prime}$ is the reverse electric field of evaporation of the same domain walls, which were nucleated by the electric field $E$.

The lines parallel to $\mathrm{CD}$ in figure $1 \mathrm{a}$ were evaporated by the electric field $5 \mathrm{kV} \mathrm{cm}^{-1}$, indicating that they were nucleated by the opposite field less than $3.3 \mathrm{kV} \mathrm{cm}^{-1}$ by a similar cooperative manner as explained above. Since no electric field was applied before to get the photomicrograph shown in figure 1a, the required electric field was assumed to be present due to the surface charge developed while cooling the crystals in the process of crystal growth just below the Curie transition temperature. The corresponding critical length will be more than $0.24 \mu \mathrm{m}$ for such domain structures. 


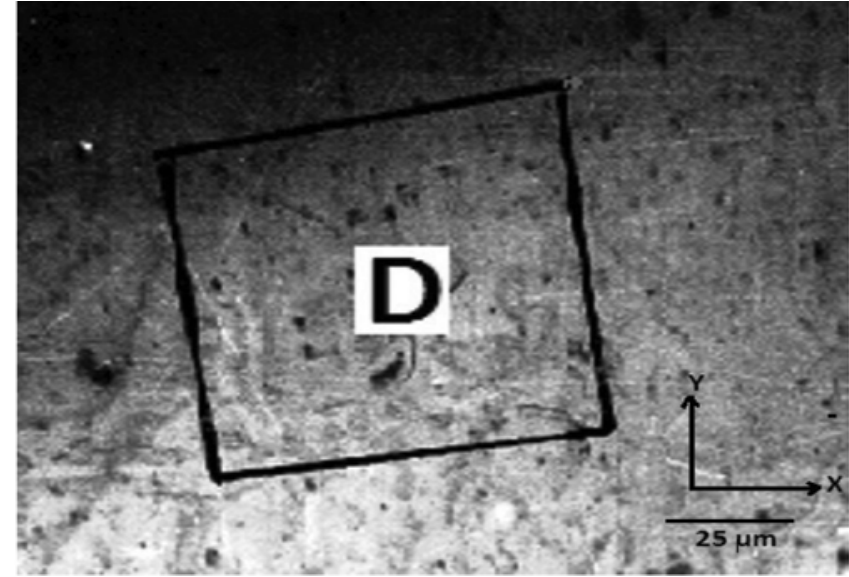

Figure 5. Photomicrograph of crystal surface showing $180^{\circ}$ domain site marked by $\mathrm{D}(560 \times)$.

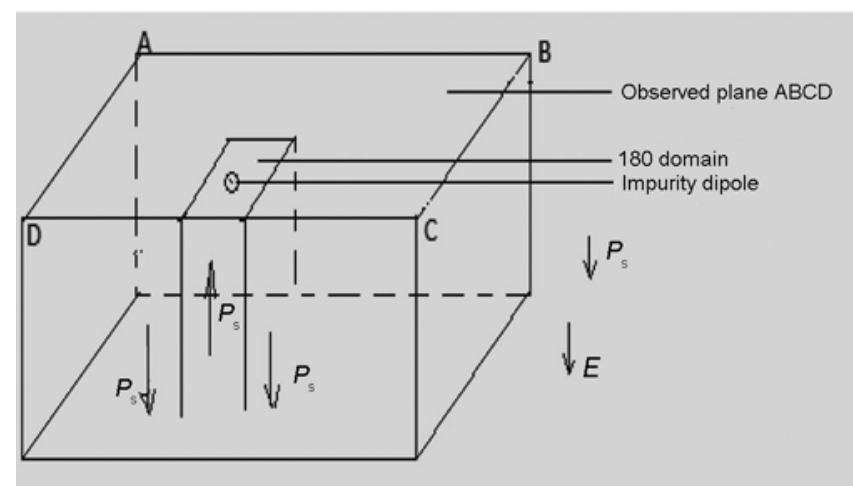

Figure 6. Schematic representation of $180^{\circ}$ domain nucleation.

The inverse relation between the electric field and the critical length of domains suggests that the naturally occurred larger stable domains are due to the presence of smaller electric field, which is usually present just below the Curie temperature during paraelectric-ferroelectric phase transition. During transition, of course processes of nucleation and evaporation of domain walls are operative even in the absence of external electric field and finally a stable domain structures are obtained at room temperature. For smaller and smaller domain structure say of the order of nm, higher and higher electric field is required which might not be present during the phase transition when the size of the crystal is very small.

\subsection{Nucleation and evaporation of the $180^{\circ}$ domains}

Hydrochloric acid is a very good etching agent which reveals $180^{\circ}$ domains of $\mathrm{BaTiO}_{3} .^{20}$ The etched surface of the crystal flake by hydrochloric acid and photograph of crystal surface are shown in figure 5 . The figure shows a major pseudocubic (001) surface of the flake. The D marked region enclosed in a square is a site of the $180^{\circ}$ domain. Schematically this is shown in figure 6. After
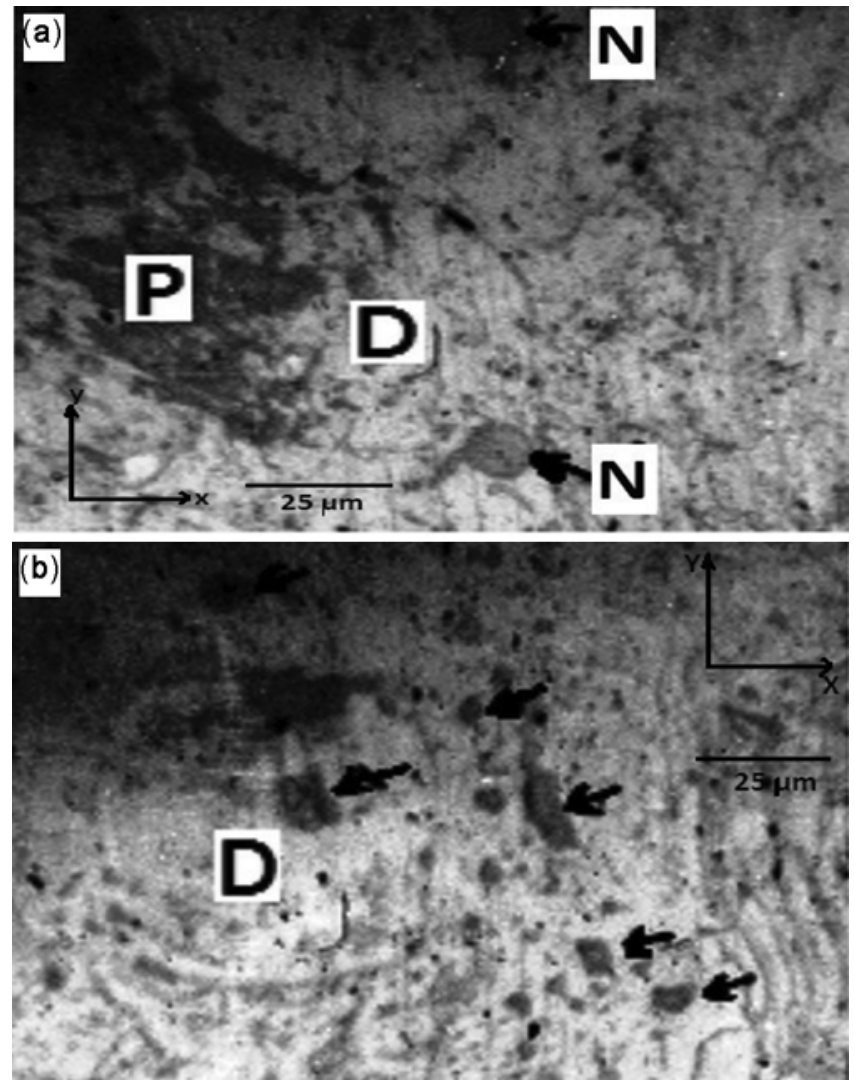

Figure 7. (a) Photomicrograph of same surface (in figure 5) after application of dc field $4 \mathrm{kV} \mathrm{cm}^{-1}$ for $10 \mathrm{~min}$, etching by hydrochloric acid for $10 \mathrm{~s}$ and washing with methanol, the figure shows new $180^{\circ}$ domains marked by $\mathrm{P}$ and $\mathrm{N}(560 \times)$ and (b) photomicrograph of same surface (in a) after application of dc field $7 \mathrm{kV} \mathrm{cm}^{-1}$ for $10 \mathrm{~min}$, reverse direction, and after etching by hydrochloric acid for $10 \mathrm{~s}$ and washing with methanol showing evaporation of previously nucleated $180^{\circ}$ domains and nucleation of another new domains marked by arrows $(560 \times)$.

application of the electric field of about $4 \mathrm{kV} \mathrm{cm}^{-1}$ for $10 \mathrm{~min}$ to its major surface, subsequently etched by hydrochloric acid for $10 \mathrm{~s}$ and washed with methanol is shown in figure $7 \mathrm{a}$. Comparison of this figure with figure 5 reveals that the earlier domain structure is altered. This photomicrograph as shown in figure 7a shows two black structures marked by $\mathrm{N}$ and black aggregates marked by $\mathrm{P}$. These structures and aggregates are not impurity clusters; if they were, then they would have been washed out by methanol, since methanol acts as a cleaning agent and removes impurity clusters from the surfaces. ${ }^{21}$ This suggests that these areas marked by $\mathrm{N}$ and $\mathrm{P}$ correspond to the $180^{\circ}$ domains.

Photomicrograph shown in figure $7 \mathrm{~b}$ is again of the same surface as referred in figure 7 a but now with the electric field of about $7 \mathrm{kV} \mathrm{cm}^{-1}$ applied in a reverse direction for $10 \mathrm{~min}$. In this photomicrograph, previously nucleated $\mathrm{N}$ and $\mathrm{P}$ marked structures, observed with the forward field, disappeared and new structure marked by arrows are appeared. 
This nucleation and evaporation of the $180^{\circ}$ domains corresponding to appearance and disappearance of the structures as seen in figures $7 \mathrm{a}$ and $\mathrm{b}$ can be explained on similar line as $90^{\circ}$ domain's nucleation and evaporation. The explanation for the mechanism involved in nucleation and evaporation of $180^{\circ}$ domains then would be as follows: impurity ions are activated and impurity dipoles are formed because of the applied dc electric field. These impurity dipoles tend to align along the upward and/or downward directions giving rise to $P_{\mathrm{s}}$ at that site, opposite to the direction of spontaneous polarization of the crystal as a whole. Consequently, a strain site is resulted and, in order to reduce this strain, $180^{\circ}$ domains are nucleated. Marked regions $\mathrm{P}$ and $\mathrm{N}$, in figure $7 \mathrm{a}$, are therefore newly nucleated $180^{\circ}$ domains due to initial forward $\mathrm{dc}$ electric field. Conversely, if the reverse field is applied then the impurity dipoles in the nucleated $180^{\circ}$ domains might have changed their orientation, deactivated and changed into ionic impurity form. This mechanism corresponds to evaporation of domains. Disappearance of structure $\mathrm{N}$ and $\mathrm{P}$ in figure $7 \mathrm{~b}$ confirms the evaporation of $180^{\circ}$ domains due to the reversed field. However, some fresh, small $180^{\circ}$ domains are seen to be appeared (marked by arrows in figure $7 \mathrm{~b}$ ), which again confirms the simultaneous nucleation and evaporation processes of $180^{\circ}$ domains in $\mathrm{BaTiO}_{3}$.

Despite exact relationship of critical length of $180^{\circ}$ domains boundary and the applied electric field, it is expected that these quantities are inversely proportional, similar to $90^{\circ}$ domains. The higher is the applied electric field, lower is the sizes of $180^{\circ}$ domains. The domain sizes in figure $7 \mathrm{a}$ and $\mathrm{b}$ are 4 and $7 \mathrm{kV} \mathrm{cm}^{-1}$, respectively, when compared this fact is supported, and also goes along with the observations of Stadler and Zachmanidis. ${ }^{22,23}$

\section{Conclusion}

Both the nucleation and evaporation of $90^{\circ}$ and $180^{\circ}$ domains are operative in $\mathrm{BaTiO}_{3}$ at room temperature under the influence of dc electric field, similar to that reported in the literature, just below the Curie temperature in the absence of external electric field. The nucleation of domains is mainly due to impurity dipoles as they can be excited easily by the electric field. Bigger domain will be formed when the dipoles concerning to such domains have head-to-tail cooperative arrangement. On the other hand, some of the domains which experiences noncooperative reverse field get evaporated. After evaporation of domains, the dipoles related to such domains will be relaxed in to ionic form.

\section{References}

1. Lines M E and Glass A M 1977 Principles and applications of ferroelectrics and related materials (Oxford: Clarendon)

2. Whatmore R 2007 Ferroelectric materials, handbook of electronic and photonic materials (ed.) S Kasap and P Capper (Heidelberg: Springer)

3. Tayebi N and Zhang Y 2011 Ultrahigh density probebased storage using ferroelectric thin films, ferroelectric applications (ed.) M Lallart (Rijeka, Croatia: InTech)

4. Megaw H D 1957 Ferroelectricity in crystals (London: Methuen)

5. Forsbergh P W Jr 1949 Phys. Rev. 761187

6. Ingle S G and Joshi S C 1986 Phys. Rev. B 344840

7. Shackelford J F and Holman R L 1975 J. Appl. Phys. 46 1429

8. Ingle S G and Moon K S 1991 J. Phys. D: Appl. Phys. 24 1637

9. Zhang Q and Goddard W A III 2006 Appl. Phys. Lett. 89 182903

10. Tanaka M and Honjo G 1964 J. Phys. Soc. Japan 19954

11. Bradt R C and Ansell G S 1967 J. Appl. Phys. 385407

12. Ingle S G, Moon K S and Kakade R N 1992 Bull. Mater. Sci. 15251

13. Potnis P R, Tsou N-T and Huber J E 2011 Materials 4417

14. Dudhe C M, Nagdeote S B, Khambadkar S J, Arjunwadkar P R and Patil R R 2014 Ferroelectrics 471148

15. Ingle S G, Dutta H S and David A P 1988 J. Appl. Phys. 64 4640

16. Ingle S G and Kokate M V 1990 Philos. Mag. A61 543

17. Ingle S G and Moon K S 1991 Ferroelectrics 119107

18. He D Y, Qiao L J, Volinsky A A, Bai Y and Guo L Q 2011 Phys. Rev. B 84024101

19. Jona F and Shirane G 1962 Ferroelectric crystals (New York: Pergamon Press)

20. Hooton J A and Merz W J 1955 Phys. Rev. 98409

21. Moon K S 1992 Role of impurity dipoles in domain formation in $\mathrm{KNbO}_{3}$ single crystals $\mathrm{PhD}$ thesis (India: Nagpur University)

22. Stadler H L and Zachmanidis 1964 J. Appl. Phys. 352895

23. Stadler H L and Zachmanidis 1964 J. Appl. Phys. 352625 